\title{
A comparative study between resting-state fMRI and plasma markers in subjects with mild cognitive impairment, on residents of Wuxi City, China.
}

teng wang ${ }^{1}$, qing $\operatorname{Lin}^{2}$, dong wang ${ }^{3}$, huo cheng ${ }^{4}$, and fu zhao ${ }^{4}$

${ }^{1}$ Wuxi Mental Health Center

${ }^{2}$ Nanjing Medical University, Jiangsu Province,China

${ }^{3}$ Suzhou GuangJi Hospital,

${ }^{4}$ Wuxi Mental Health Centre

August 19, 2020

\begin{abstract}
From April 2018 to December 2019, we recruited 31 volunteers with mild cognitive impairment and 33 volunteers in the healthy control group from the residents of Wuxi City's community. Relevant researches on resting state functional magnetic resonance and human plasma markers were done respectively; Studies have shown that there are differences in brain function between the MCI group and the healthy control group, which is similar to previous studies[1]; It is worth noting that most plasma markers and cerebrospinal fluid, apolipoprotein gene E have no significant difference, Most previous studies of the same kind have shown anomalies[2], and we speculate that this difference may be related to ethnic or regional differences[3, 4]. Next, we will expand the sample size to further verify this guess.
\end{abstract}

\section{Hosted file}

\begin\{CJK\}\{UTF8\}\{gbsn\}. \end\{CJK\}\selectlanguage\{english\}doc available at https://authorea. } com/users/348515/articles/476495-a-comparative-study-between-resting-state-fmri-andplasma-markers-in-subjects-with-mild-cognitive-impairment-on-residents-of-wuxi-citychina

\section{Hosted file}

$2 \backslash$ begin $\{\mathrm{CJK}\}\{\mathrm{UTF} 8\}\{\mathrm{gbsn}\}$. \end\{CJK\}\selectlanguage\{english\}docx available at https: } //authorea.com/users/348515/articles/476495-a-comparative-study-between-resting-statefmri-and-plasma-markers-in-subjects-with-mild-cognitive-impairment-on-residents-of-wuxicity-china

\section{Hosted file}

3\begin\{CJK\}\{UTF8\}\{gbsn\}. \end\{CJK\}\selectlanguage\{english\}docx available at https: } //authorea.com/users/348515/articles/476495-a-comparative-study-between-resting-statefmri-and-plasma-markers-in-subjects-with-mild-cognitive-impairment-on-residents-of-wuxicity-china

\section{Hosted file}

table.doc available at https://authorea.com/users/348515/articles/476495-a-comparativestudy-between-resting-state-fmri-and-plasma-markers-in-subjects-with-mild-cognitive- 
impairment-on-residents-of-wuxi-city-china 